\title{
Feasibility of Hand-Assisted Laparoscopic Surgery as Compared to Open Surgery for Sigmoid Colon Cancer: A Case-Controlled Study
}

\author{
Sang Eun Nam, Eun-Joo Jung, Chun-Geun Ryu, Jin Hee Paik, Dae-Yong Hwang \\ Department of Surgery, Colorectal Cancer Center, Konkuk University Medical Center, Konkuk University School of Medicine, Seoul, Korea
}

Purpose: The aim of this study was to evaluate short-term clinical outcomes by comparing hand-assisted laparoscopic surgery (HALS) with open surgery for sigmoid colon cancer.

Methods: Twenty-six patients who underwent a hand-assisted laparoscopic anterior resection (HAL-AR group) and 52 patients who underwent a conventional open anterior resection during the same period were enrolled (open group) in this study with a case-controlled design.

Results: Pathologic parameters were similar between the two groups. The incidences of immediate postoperative leukocytosis were $38.5 \%$ in the HAL-AR group and $69.2 \%$ in the open group $(\mathrm{P}=0.009)$. There were no significant differences between the two groups as to leukocyte count, hemoglobin, and hematocrits $(\mathrm{P}=0.758, \mathrm{P}=0.383$, and $\mathrm{P}=0.285$, respectively). Of the postoperative recovery indicators, first flatus, sips of water and soft diet started on postoperative days $3,5,7$ in the HALS group and on days 4, 5, 6 in the open group showed statistical significance $(\mathrm{P}=0.021, \mathrm{P}=0.259$, and $\mathrm{P}=0.174$, respectively). Administration of additional pain killers was needed for 1.2 days in the HAL-AR group and 2.4 days in the open group $(\mathrm{P}=$ 0.002). No significant differences in the durations of hospital stay and the rates of postoperative complications were noted, and no postoperative mortality was encountered in either group.

Conclusion: The patients with sigmoid colon cancer who underwent a HAL-AR had a lower incidence of postoperative leukocytosis, less administration of pain killers, and faster first flatus than those who underwent open surgery. Clinical outcomes for patients' recovery and pathology status were similar between the two groups. Therefore, a HAL-AR for sigmoid colon cancer is feasible and has the same benefit as minimally invasive surgery.

Keywords: Hand-assisted laparoscopic surgery; Minimally invasive surgery; Anterior resection; Sigmoid colon cancer

\section{INTRODUCTION}

Since laparoscopic colorectal surgery was introduced in the early 1990s, minimally invasive surgery for colorectal diseases has been

Received: January 31, 2013 • Accepted: February 15, 2013

Correspondence to: Dae-Yong Hwang, M.D.

Department of Surgery, Colorectal Cancer Center, Konkuk University Medical Center, Konkuk University School of Medicine, 120 Neungdong-ro,

Gwangjin-gu, Seoul 143-729, Korea

Tel: +82-2-2030-5111, Fax: +82-2-2030-5112

E-mail: hwangcrc@kuh.ac.kr

This was presented at 64th Annual Congress of the Korean Surgical Society.

(c) 2013 The Korean Society of Coloproctology

This is an open-access article distributed under the terms of the Creative Commons Attribution NonCommercial License (http://creativecommons.org/licenses/by-nc/3.0) which permits unrestricted noncommercial use, distribution, and reproduction in any medium, provided the original work is properly cited. extended with the development of new instruments [1-4]. Laparoscopic surgery has many advantages, such as smaller incision, less pain, faster postoperative recovery, and shorter hospital stay; however, limitations, such as its complexity, long operation time, long learning curve and lack of tactile feedback, still exist [1].

Hand-assisted laparoscopic surgery (HALS) originated from using a mini-laparotomy for specimen retrieval or an extracorporeal procedure in pure laparoscopic surgery [2]. This procedure is a kind of minimally invasive surgery, but has the features of open surgery [2]. The most remarkable factor of HALS is that the operator's hand can be placed into the intra-abdominal cavity. The operator's hand can palpate organs or tumors, retract structures gently, identify vessels, and provide finger pressure to bleeding points. In other words, HALS is a kind of hybrid procedure between pure laparoscopic surgery and conventional open surgery [2]. Although many studies related to the use of HALS for treating colorectal dis- 
ease have been performed, few reports comparing HALS to open surgery in patients with sigmoid colon cancer have been published. Therefore, the aim of this study was to assess the clinico-pathologic parameters and the short-term clinical outcomes of a handassisted laparoscopic anterior resection (HAL-AR) in patients with sigmoid colon cancer by comparing them to those of a conventional open anterior resection.

\section{METHODS}

Using the prospectively collected colorectal cancer database at our institute, we designed a case-controlled study. Between January 2009 and October 2011, of the patients with an adenocarcinoma on the sigmoid colon, 26 who underwent a HAL-AR (HAL-AR group) and 52 who underwent a conventional open anterior resection (open group) were enrolled as the study and the control groups, respectively. Patients in the control group were selected so as to be case-matched with those in the HAL-AR group in the aspects of age, sex, and tumor-node-metastasis (TNM) stage. All patients underwent a curative resection by one experienced surgeon. Patients with cancer obstruction or perforation were excluded, as were patients who underwent emergent surgery.

In postoperative care, patient-controlled analgesia (PCA) was applied for all patients, and additional intravenous analgesia was administrated when required. Sips of water began when patients had no discomfort in the abdomen after first flatus, and soft diet was started one day after. Patients were discharged when soft diet was tolerable and they agreed. Regular laboratory tests were performed on postoperative days 1,2, 4, and 7; especially, a postoper-

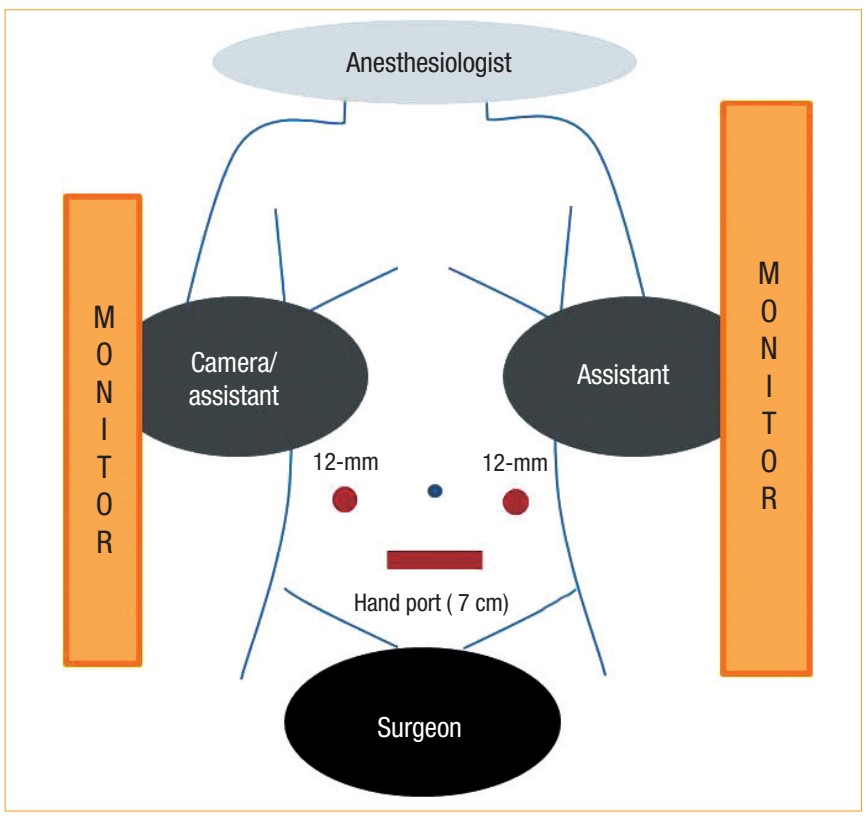

Fig. 1. Operating-room setup for hand-assisted laparoscopic anterior resection. ative white blood cell count in analysis was done on postoperative day 1 . Between the HAL-AR group and the open group, clinicopathologic, intraoperative and postoperative parameters were analyzed statistically.

\section{Surgical techniques}

Under endotracheal intubation, the patient was positioned on the surgical table in a modified lithotomy position by using Allen's stirrups, and a pneumatic compression device was applied to the patient's legs. The operative field was prepared in the usual manner and draped. The operating room setup for the HAL-AR is shown in Fig. 1.

First, a Pfannenstiel incision with a 7-cm length was made on the lower abdomen, and a Gelport (Applied Medical Resources Co., Rancho Santa Margarita, CA, USA) was applied to the skin at the incision. A Gelport is a kind of hand-port that can maintain the pneumoperitoneum while the surgeon's hand is in the abdominal cavity. On the right and the left sides of the abdomen at the level of the umbilicus, outside the rectus abdominis muscle, a $12-\mathrm{mm}$ trocar was inserted, and a pneumoperitoneum was made with $\mathrm{CO}_{2}$ gas.

Then, the surgeon, who was located between the patient's legs, inserted the laparoscopic camera via the right-side port. The surgeon's left hand was inserted into the hand-port, and the inferior mesenteric artery was isolated and ligated with clips by using a laparoscopic device inserted via the left-side port. Then, the sigmoid colon mesentery was dissected using the medial-to-lateral approach. After complete dissection of the sigmoid colon, the Gelport was removed, and next steps were conducted as in open surgery. The sigmoid colon was resected, and a specimen was delivered. Anastomosis was performed using the hand-sewn or double-stapled method. After warm saline irrigation and meticulous hemostasis, the Gelport was reapplied, and a pneumoperitoneum was made to check the anastomosis alignment and the dissection plane for bleeding. A Jackson-Pratt drain was inserted into the pelvic cavity via the left port. The right port and the Pfannenstiel incision were closed layer by layer after the Gelport had again been removed.

\section{Statistical analysis}

Data analysis was performed using the SPSS ver. 14.0 (SPSS Inc., Chicago, IL, USA). Summary statistics using the chi-squared test, the two-sample t-test with Welch's correction, and Fisher's exact test were used to compare the HAL-AR group with the open group. Statistical significance was associated with P-values less than 0.05 .

\section{RESULTS}

The total 78 cases consisted of 26 in the HAL-AR group and 52 in the open group. As to the patients' characteristics, the gender ratio (male : female) was $1.9: 1$ (17 vs. 9) in the HAL-AR group and 1.9: 1 (34 vs. 18$)$ in the open group $(\mathrm{P}=0.596)$. The mean age was 60 
Table 1. Patients' characteristics

\begin{tabular}{|c|c|c|c|}
\hline Characteristic & HAL-AR group $(n=26)$ & Open group $(n=52)$ & P-value \\
\hline Gender (M:F) & $17: 9$ & $34: 18$ & 0.596 \\
\hline Age (yr) & $60.0 \pm 12.6$ & $61.3 \pm 9.9$ & 0.465 \\
\hline Body mass index $\left(\mathrm{kg} / \mathrm{m}^{2}\right)$ & $24.6 \pm 3.2$ & $23.6 \pm 4.6$ & 0.769 \\
\hline Preoperative comorbidities & $11(42.3)$ & $36(69.2)$ & \\
\hline Hypertension & $3(11.4)$ & $8(15.4)$ & 0.067 \\
\hline Diabetes mellitus & $5(19.2)$ & $18(34.6)$ & 0.126 \\
\hline Pulmonary tuberculosis & $2(7.7)$ & $2(3.8)$ & 0.407 \\
\hline Others ${ }^{\mathrm{a}}$ & $1(3.8)$ & $8(15.4)$ & 0.127 \\
\hline Obstruction ${ }^{b}$ & $3(11.5)$ & $3(5.8)$ & 0.315 \\
\hline Incidence of preoperative carcinoembryonal antigen elevation & $2(7.7)$ & $10(19.2)$ & 0.159 \\
\hline Incidence of preoperative carbohydrate antigen 19-9 elevation & $0(0)$ & $3(5.8)$ & 0.290 \\
\hline Level of preoperative carcinoembryonal antigen (ng/mL) & $5.6 \pm 14.0$ & $4.7 \pm 7.5$ & 0.448 \\
\hline Level of preoperative carbohydrate antigen 19-9 (ng/mL) & $10.7 \pm 8.1$ & $12.0 \pm 16.7$ & 0.054 \\
\hline
\end{tabular}

Values are presented as mean \pm standard deviation or number (\%).

HAL-AR, hand-assisted laparoscopic anterior resection.

aHAL-AR group, cerebral infarction; open group, stomach cancer, atrial septal defect, valvular heart disease, cerebral infarction, congestive heart failure, chronic renal failure, and chronic liver disease. ${ }^{b}$ Preoperative colonic stent was inserted.

Table 2. Operative and pathologic parameters in the HAL-AR and the open groups

\begin{tabular}{lccc}
\hline Parameter & $\begin{array}{c}\text { HAL-AR group } \\
(\mathrm{n}=26)\end{array}$ & $\begin{array}{c}\text { Open group } \\
(\mathrm{n}=52)\end{array}$ & P-value \\
\hline Operation time (min) & $\begin{array}{c}224.4 \pm 31.7 \\
(192-256)\end{array}$ & $\begin{array}{c}195.2 \pm 66.4 \\
(129-261)\end{array}$ & 0.162 \\
Conversion case & $0(0)$ & & \\
Intraoperative complication & $0(0)$ & $0(0)$ & \\
Anastomosis method & & & 0.070 \\
$\quad$ Double stapled & 23 & 51 & \\
$\quad$ Hand-sewn & 3 & 1 & \\
TNM stage & & & 0.688 \\
0 & 11 & 16 & \\
I & 4 & 13 & \\
॥ & 9 & 18 & \\
III & $3.1 \pm 2.2$ & $3.7 \pm 4.1$ & 0.338 \\
Tumor size (cm) & $17.5 \pm 9.2$ & $15.3 \pm 7.8$ & 0.211 \\
No. of retrieved lymph node & $3(11.5)$ & $13(25.0)$ & 0.137 \\
Lymphatic invasion & $1(3.8)$ & $3(5.8)$ & 0.593 \\
Perineural invasion & $1(3.8)$ & $1(1.9)$ & 0.558 \\
\hline Venous invasion & & 5 & \\
\hline Valn & & & \\
\hline
\end{tabular}

Values are presented as mean \pm standard deviation (range) or number (\%).

HAL-AR, hand-assisted laparoscopic anterior resection; TNM, tumor-node-metastasis.

years in the HAL-AR group and 61 years in the open group $(\mathrm{P}=$ $0.465)$. No significant differences existed between the groups in
Table 3. Postoperative laboratory data and recovery parameters

\begin{tabular}{lccc}
\hline & $\begin{array}{c}\text { HAL-AR group } \\
(\mathrm{n}=26)\end{array}$ & $\begin{array}{c}\text { Open group } \\
(\mathrm{n}=52)\end{array}$ & P-value \\
\hline Leukocytosis & $10(38.5)$ & $36(69.2)$ & 0.009 \\
WBC count difference $(/ \mu \mathrm{L})^{\mathrm{a}}$ & $5,600 \pm 3,500$ & $4,300 \pm 2,800$ & 0.758 \\
Extra-pain killer use & $22(84.6)$ & $48(92.3)$ & 0.249 \\
Day of pain killer administration & $1.2 \pm 0.8$ & $2.4 \pm 3.0$ & 0.002 \\
Day to flatus (day) & $3.2 \pm 1.8$ & $3.8 \pm 1.1$ & 0.021 \\
Day to sips of water (day) & $4.9 \pm 1.8$ & $4.5 \pm 1.3$ & 0.259 \\
Day to soft diet (day) & $6.8 \pm 2.1$ & $6.2 \pm 1.5$ & 0.174 \\
Day to discharge (day) & $11.7 \pm 3.8$ & $11.5 \pm 4.0$ & 0.923 \\
Postoperative complication & $0(0)$ & $1(1.9)^{\mathrm{b}}$ & 0.667 \\
Readmission & $0(0)$ & $0(0)$ & \\
Reoperation & $0(0)$ & $0(0)$ & \\
Mortality & $0(0)$ & $0(0)$ & \\
\hline
\end{tabular}

Values are presented as number (\%) or mean \pm standard deviation.

HAL-AR, hand-assisted laparoscopic anterior resection; WBC, white blood cell. aPostoperative WBC count-preoperative WBC count. ${ }^{b}$ Postoperative ileus problem.

terms of gender, age, comorbidity, body mass index, obstruction, or tumor markers (Table 1).

The mean operation time was 224 minutes in the HAL-AR group and 195 minutes in the open group $(\mathrm{P}=0.162)$. The operation time in the open group was slightly, but not significantly, shorter than it was in the HAL-AR group. No conversion to open surgery was needed in the HAL-AR group, and no intraoperative compli- 
cations were encountered in either group. In most cases, the colonic anastomosis was performed mainly using the double-stapled method. Between the two groups, no significant differences were noted in either the distribution of TNM stages or pathologic factors, such as tumor size, number of retrieved lymph nodes, and lymphovascular or neural invasion (Table 2).

The postoperative parameters for the two groups are shown in Table 3. The incidence of immediate postoperative leukocytosis in the HAL-AR group (38.5\%) was significantly lower than it was in the open group $(69.2 \%)(\mathrm{P}=0.009)$. No significant differences were noted between the two groups in regard to leukocyte count, hemoglobin and hematocrits $(\mathrm{P}=0.758, \mathrm{P}=0.383$, and $\mathrm{P}=0.285$, respectively). Compared to patients in the open group, those in the HAL-AR group used significantly fewer pain killers and experienced earlier passage of flatus after surgery. Administration of additional pain killers was needed for 1.2 days in the HAL-AR group and 2.4 days in the open group $(\mathrm{P}=0.002)$. No significant differences in hospital stay (11.7 days vs. 11.5 days, $\mathrm{P}=0.923)$ and postoperative complications ( 0 vs. $1, \mathrm{P}=0.667$ ) were noted. The one complication in the open surgery group was postoperative ileus, and that patient recovered after conservative management. No postoperative mortality or reoperation occurred in either group.

\section{DISCUSSION}

According to our data, compared to the patients in the conventional open anterior resection group, those who received a HALAR for sigmoid colon cancer experienced the following advantages: a lower incidence of postoperative leukocytosis, less administration of pain killers and faster recovery of gastrointestinal function. These factors mean less surgical trauma, less pain and faster recovery, which are also the advantages of minimally invasive surgery.

According to some studies comparing open surgery with HALS for treating colorectal diseases, a common conclusion was that HALS had a smaller incision, a faster postoperative recovery and a shorter hospital stay [5-9]. Our data showed similar results: small incisions, reductions in the amounts of additional pain killers required, and fast recovery of bowel movement. In addition, the complication rates, as well as the incidences of reoperations, readmissions and mortalities, were comparable between the two groups. However, the duration of hospital stay was similar, about 11 days, in both groups, which was probably because the patients tended to stay a few days longer even though they were on a tolerable diet and in a general condition acceptable for discharge.

Our data also demonstrated similar operation times between the two groups. Most previous studies had demonstrated that the operation time was significantly longer in the HALS group than in the open surgery group $[7,9,10]$. In those studies, the surgeon's experience was not mentioned; neither did those studies address whether multiple surgeons had performed various types operations, e.g., anterior resections, right or left hemicolectomies, low anterior resections or total colectomies. In contrast, this study enrolled only those patients who underwent an anterior resection performed by one surgeon with sufficient experience with open colorectal surgery [3,7,9,11-15]. Kang et al. [6] also reported similar operation times for both the HALS and the open surgery groups when the surgery was conducted by one surgeon, which implies that the longer operation time in the HALS group is most likely due to the surgeon's lack of experience, not the type of operation.

In other studies comparing HALS with conventional laparoscopic surgery for treating colorectal diseases [3,4,16-18], the postoperative clinical outcomes were similar between the two groups; in addition, the rate of conversion to open surgery was lower in the HALS group than in the conventional laparoscopic surgery group $[4,16,17,19,20]$. On the other hand, HALS patients had a rather long incision length and more severe surgical trauma than laparoscopic-surgery patients $[4,16,17,19,20]$. Nevertheless, the benefits of HALS countervail the demerits; that is, HALS had a reduced open conversion rate, the surgeon's hand maintained tactile sensation $[2,17,20-22]$. Also, when an unexpected or serious situation occurred during surgery, the surgeon could approach the operative field directly and solve the problem quickly when using HALS technique [2,17,20-22].

Minimally invasive surgery for treating colorectal diseases has already been reported to be safe and technically feasible $[5,16,19]$. However, most studies included diverse categories of colorectal diseases, including diverticulitis, inflammatory bowel diseases, sigmoid volvulus, colorectal cancer and so on $[8,9,12,13]$. In the case of malignant disease, not only the feasibility of surgery, but also oncologic safety should be considered and evaluated. Thus, the number of retrieved lymph nodes should be one parameter describing the extent of lymph-node dissection. Our data demonstrated that the numbers of retrieved lymph nodes were 17.5 in the HAL-AR group and 15.3 in the open group, being more than 12 in both group [23]. Sheng et al. [8] reported no significant difference in the numbers of retrieved lymph nodes between patients who underwent a hand-assisted laparoscopic right hemicolectomy for the treatment of right colon cancer and those who underwent an open right hemicolectomy. However, there are few reports on the use of an anterior resection for the treatment of sigmoid colon cancer.

As described in the introduction, hand-assisted laparoscopic colorectal surgery is a hybrid technique, which has the merits of both laparoscopic and conventional open surgery for the treatment of sigmoid colon cancer, even though some surgeons considered HALS to be a bridge from open surgery to laparoscopic surgery.

In conclusion, patients with sigmoid colon cancer who underwent a HAL-AR had not only a lower incidence of postoperative leukocytosis, less administration of pain killers, and faster recovery of the gastrointestinal tract, but also a radicality of lymph-node dissection similar to that in open cases. Therefore, the use of a 
HAL-AR for the treatment of sigmoid colon cancer is feasible and safe and can be considered as an alternative procedure to open surgery.

\section{CONFLICT OF INTEREST}

No potential conflict of interest relevant to this article was reported.

\section{REFERENCES}

1. Cima RR, Pendlimari R, Holubar SD, Pattana-Arun J, Larson DW, Dozois EJ, et al. Utility and short-term outcomes of hand-assisted laparoscopic colorectal surgery: a single-institution experience in 1103 patients. Dis Colon Rectum 2011;54:1076-81.

2. Darzi A. Hand-assisted laparoscopic colorectal surgery. Surg Endosc 2000;14:999-1004.

3. Datta V, Bann S, Hernandez J, Darzi A. Objective assessment comparing hand-assisted and conventional laparoscopic surgery. Surg Endosc 2007;21:414-7.

4. Marcello PW, Fleshman JW, Milsom JW, Read TE, Arnell TD, Birnbaum EH, et al. Hand-assisted laparoscopic vs. laparoscopic colorectal surgery: a multicenter, prospective, randomized trial. Dis Colon Rectum 2008;51:818-26.

5. Aalbers AG, Doeksen A, Van Berge Henegouwen MI, Bemelman WA. Hand-assisted laparoscopic versus open approach in colorectal surgery: a systematic review. Colorectal Dis 2010;12:287-95.

6. Kang JC, Chung MH, Chao PC, Yeh CC, Hsiao CW, Lee TY, et al. Hand-assisted laparoscopic colectomy vs open colectomy: a prospective randomized study. Surg Endosc 2004;18:577-81.

7. Liu FL, Lin JJ, Ye F, Teng LS. Hand-assisted laparoscopic surgery versus the open approach in curative resection of rectal cancer. J Int Med Res 2010;38:916-22.

8. Sheng QS, Lin JJ, Chen WB, Liu FL, Xu XM, Lin CZ, et al. Handassisted laparoscopic versus open right hemicolectomy: short-term outcomes in a single institution from China. Surg Laparosc Endosc Percutan Tech 2012;22:267-71.

9. Orenstein SB, Elliott HL, Reines LA, Novitsky YW. Advantages of the hand-assisted versus the open approach to elective colectomies. Surg Endosc 2011;25:1364-8.

10. Chiu CC. Letter 1: Short-term outcomes from a prospective randomized trial comparing laparoscopic and open surgery for colorectal cancer (Br J Surg 2009; 96: 1458-1467). Br J Surg 2010; 97;789.

11. Ozturk E, da Luz Moreira A, Vogel JD. Hand-assisted laparoscop- ic colectomy: the learning curve is for operative speed, not for quality. Colorectal Dis 2010;12(10 Online):e304-9.

12. Pendlimari R, Touzios JG, Azodo IA, Chua HK, Dozois EJ, Cima $\mathrm{RR}$, et al. Short-term outcomes after elective minimally invasive colectomy for diverticulitis. Br J Surg 2011;98:431-5.

13. Romanelli JR, Kelly JJ, Litwin DE. Hand-assisted laparoscopic surgery in the United States: an overview. Semin Laparosc Surg 2001;8:96-103.

14. Meshikhes AW, El Tair M, Al Ghazal T. Hand-assisted laparoscopic colorectal surgery: initial experience of a single surgeon. Saudi J Gastroenterol 2011;17:16-9.

15. Ballantyne GH, Leahy PF. Hand-assisted laparoscopic colectomy: evolution to a clinically useful technique. Dis Colon Rectum 2004;47:753-65.

16. HALS Study Group. Hand-assisted laparoscopic surgery vs standard laparoscopic surgery for colorectal disease: a prospective randomized trial. Surg Endosc 2000;14:896-901.

17. Targarona EM, Gracia E, Garriga J, Martinez-Bru C, Cortes M, Boluda R, et al. Prospective randomized trial comparing conventional laparoscopic colectomy with hand-assisted laparoscopic colectomy: applicability, immediate clinical outcome, inflammatory response, and cost. Surg Endosc 2002;16:234-9.

18. Yun HR, Cho YK, Cho YB, Kim HC, Yun SH, Lee WY, et al. Comparison and short-term outcomes between hand-assisted laparoscopic surgery and conventional laparoscopic surgery for anterior resections of left-sided colon cancer. Int J Colorectal Dis 2010;25: 975-81.

19. Marcello PW. Hand-assisted laparoscopic colectomy: a helping hand? Clin Colon Rectal Surg 2004;17:125-9.

20. Hassan I, You YN, Cima RR, Larson DW, Dozois EJ, Barnes SA, et al. Hand-assisted versus laparoscopic-assisted colorectal surgery: practice patterns and clinical outcomes in a minimally-invasive colorectal practice. Surg Endosc 2008;22:739-43.

21. Southern Surgeons' Club Study Group. Handoscopic surgery: a prospective multicenter trial of a minimally invasive technique for complex abdominal surgery. Arch Surg 1999;134:477-85.

22. Darzi A. Hand-assisted laparoscopic colorectal surgery. Semin Laparosc Surg 2001;8:153-60.

23. National Comprehensive Cancer Network. NCCN clinical practice guidelines in oncology (NCCN Guidelines): colon/rectal cancer. ver. 1. 2010 [Internet]. Fort Wathington: NCCN; c2012 [cited 2013 Jan 15]. Available from: http://www.nccn.org/professionals/physician_gls/f_guidelines.asp. 